Research Paper

\title{
SET oncoprotein accumulation regulates transcription through DNA demethylation and histone hypoacetylation
}

\author{
Luciana O. Almeida ${ }^{1,3}$, Marinaldo P.C. Neto ${ }^{1}$, Lucas O. Sousa ${ }^{1}$, Maryna A. Tannous ${ }^{1}$, \\ Carlos Curti' ${ }^{2}$, Andreia M. Leopoldino ${ }^{1,4}$ \\ ${ }^{1}$ Department of Clinical Analyses, Toxicology and Food Sciences, School of Pharmaceutical Sciences of Ribeirão Preto, \\ University of São Paulo, Ribeirão Preto, SP, Brazil \\ ${ }^{2}$ Department of Physics and Chemistry, School of Pharmaceutical Sciences of Ribeirão Preto, University of São Paulo, Ribeirão \\ Preto, SP, Brazil \\ ${ }^{3}$ Department of Genetics and Evolution, Federal University of São Carlos, São Carlos, SP, Brazil \\ ${ }^{4}$ CEPID-FAPESP, Center for Cell Based Therapy, Hemotherapy Center of Ribeirão Preto, Ribeirão Preto, SP, Brazil
}

Correspondence to: Andreia M. Leopoldino, email: andreiaml@usp.br

Keywords: chromatin, HNSCC, SET protein, histone acetylation, active DNA demethylation

Received: June 30, 2016

Accepted: February 20, 2017

Published: March 01, 2017

Copyright: Almeida et al. This is an open-access article distributed under the terms of the Creative Commons Attribution License (CC-BY), which permits unrestricted use, distribution, and reproduction in any medium, provided the original author and source are credited.

\section{ABSTRACT}

Epigenetic modifications are essential in the control of normal cellular processes and cancer development. DNA methylation and histone acetylation are major epigenetic modifications involved in gene transcription and abnormal events driving the oncogenic process. SET protein accumulates in many cancer types, including head and neck squamous cell carcinoma (HNSCC); SET is a member of the INHAT complex that inhibits gene transcription associating with histones and preventing their acetylation. We explored how SET protein accumulation impacts on the regulation of gene expression, focusing on DNA methylation and histone acetylation. DNA methylation profile of 24 tumour suppressors evidenced that SET accumulation decreased DNA methylation in association with loss of 5-methylcytidine, formation of 5-hydroxymethylcytosine and increased TET1 levels, indicating an active DNA demethylation mechanism. However, the expression of some suppressor genes was lowered in cells with high SET levels, suggesting that loss of methylation is not the main mechanism modulating gene expression. SET accumulation also downregulated the expression of $\mathbf{3 2}$ genes of a panel of 84 transcription factors, and SET directly interacted with chromatin at the promoter of the downregulated genes, decreasing histone acetylation. Gene expression analysis after cell treatment with 5-aza-2'-deoxycytidine (5-AZA) and Trichostatin A (TSA) revealed that histone acetylation reversed transcription repression promoted by SET. These results suggest a new function for SET in the regulation of chromatin dynamics. In addition, TSA diminished both SET protein levels and SET capability to bind to gene promoter, suggesting that administration of epigenetic modifier agents could be efficient to reverse SET phenotype in cancer

\section{INTRODUCTION}

Epigenetics refers to heritable changes in gene expression not related to changes in the DNA sequence [1]; they occur primarily through DNA methylation and histone post-translational modifications. Epigenetic changes have been associated with specific patterns of gene expression and are early events in different cancer types, including head and neck squamous cell carcinoma (HNSCC) [2]. In mammals, DNA methylation occurs predominantly at $\mathrm{CpG}$ dinucleotides. DNA methyl transferase (DNMT) transfers a methyl group to cytosine, generating 5-methylcytosine [3]. Low methylation levels within the promoter region have been associated with either gene activation or chromosome instability [4]. 
Lack or reduction of DNMT activity implies gradual and passive loss of DNA methylation, so that DNMTs inhibition is an important mechanism of the passive DNA demethylation process. DNA demethylation also occurs through an active mechanism, independent of cell division, which is mediated by enzymes of the TET family. These enzymes firstly modify methylated cytosine, which is subsequently replaced by the DNA repair machinery $[5,6]$.

Changes in chromatin dynamics is an early abnormality described in cancer [7]. The basic unit of chromatin is the nucleosome, which is formed by DNA and histone proteins [8]. Histones undergo many modifications in their N-terminal tails, among which lysine acetylation is the most studied one. Histone deacetylases (HDAC) remove the acetyl radical present in the lysine residues of histones, whereas histone acetyltransferases (HAT) add acetyl radicals to those positions [1]. The chromatin organization results from post-translational modifications of histone tails, DNA methylation patterns, and nucleosome positioning. In cancer cells, chromatin remodeling, methylation signatures, and consequently, gene expression profiles, become disturbed [7].

SET oncoprotein (I2PP2A or TAF-1 $\beta$ ), which is accumulated in HNSCC [9], is involved in many aspects related to chromatin structure. SET protein is characterized as $(i)$ an histone chaperone required for chromatin transcription $[10,11]$, (ii) an important regulator of chromosome condensation [12], (iii) a member of the Inhibitor of Histone Acetyltransferase (INHAT) complex that inhibits histone acetylation by binding to and masking histone acetyltransferases targets [13], and (iv) an inhibitor of active DNA demethylation [14]. In this context, it is unknown whether SET accumulation in cells either modifies specific targets or is associated with transcriptional repression or activation. Here, we addressed this issue. Overall, we demonstrated that SET promotes a global DNA demethylation in HNSCC cells, through active signaling, increasing TET1 and 5-hydroxymethylcytosine levels. Demethylation is not always followed by reactivation of gene expression.

\section{RESULTS}

\section{SET accumulation is associated with global loss of DNA methylation in HNSCC}

To investigate how SET protein accumulation influences the epigenetic control of gene expression, we analysed the DNA methylation profile in two non-tumour cell lines, HEK293 and NOK-SI, with and without SET overexpression (HEK293/SET and NOK-SI/SET, respectively), and three HNSCC cell lines, HN6, HN12, and HN13, which present SET constitutively accumulated [15], with and without SET knockdown (HN6siSET, HN12siSET, and HN13siSET, respectively). As the oncogene SET is a potent inhibitor of the tumour suppressor PP2A [16, 17], and inhibits other tumour suppressors such as PTEN [18] and TP53 [19], we examined how SET accumulation affects DNA methylation using a panel of known tumour suppressor genes by means of EpiTect Methyl qPCR arrays (Figure 1A). Notably, SET protein accumulation, a common event in HNSCC [15], promoted loss of DNA methylation (Figure 1A - black arrows). Next, we analysed the average methylation change to know how much the changes in SET levels would influence the methylation profile. We calculated the ratio of $\mathrm{CpGs}$ methylated between control and up- or downregulated SET samples (Figure 1B). SET-accumulating cells displayed decreased average DNA methylation (Figure 1B - red arrows), while the HNSCC cell lines with knocked down SET displayed increased average DNA methylation (Figure 1B - green arrows); these findings suggest that SET accumulation influences DNA methylation.

To confirm these results, we analysed the cellular levels of 5-methylcytidine, which is commonly used to assess the full genome methylation profile [20]. We selected the HN12 cell line - which exhibited the most marked change in average DNA methylation after SET knockdown (Figure 1B) - and the non-tumour cell line HEK293 (Figure 1C) for the assay. Compared with HEK293 cells, HEK293/SET cells significantly lost 5-methylcytidine, while HN12 cells exhibited low 5-methylcytidine levels. As expected, HN12siSET cells had increased 5-methylcytidine levels. These results indicate that SET modulates DNA methylation levels in HNSCC.

Given that DNA methylation is an epigenetic mechanism for silencing gene expression, we performed qPCR to assess the expression levels of four genes that were hypomethylated by SET accumulation: BRCA1, GSTP1, MLH1, and PTEN (Figure 2A). They were analysed in HEK293, HN12, and HN13 cells. SET overexpression in HEK293 cells upregulated BRCA1 and MLH1 expression, but downregulated GSTP1 and PTEN expression (Figure 2A - HEK293 column). Compared with the basal expression levels in the non-tumour cell line HEK293, used as calibrator, the expression levels of all four genes were lowered in HN12 and HN13 tumour cells (Figure 2A - red bars) but increased after SET knockdown (Figure 2A - green bars). Thus, high SET levels in tumour cells directly correlated with diminished expression of these genes. This finding was unexpected because SET accumulation is associated with loss of DNA methylation, which activates the expression of genes that are typically silenced by methylation [21]. A plausible explanation is that DNA methylation responds differently to the gene expression control machinery due to the interaction with other factors, including histone modifiers [22]. In addition, SET negatively regulates the expression of genes involved in cellular detoxification [23] and also participates as a subunit of the inhibitor of histone acetyltransferases 
complex that represses transcription [24], suggesting that the SET controls gene expression through a DNA methylation-independent mechanism. Considering that SET, as a member of the INHAT complex, can directly bind to histones at promoters, to prevent acetylation $[13,24]$, we assessed the SET-promoter interaction using the chromatin immunoprecipitation assay (ChIP) for two genes whose expressions were decreased by SET accumulation: GSTP1 and PTEN (Figure 2A). Both genes demonstrated association with SET at the promoter in all SET-accumulating cells, but not in HEK293 cells, which do not accumulate SET (Figure 2B).
A

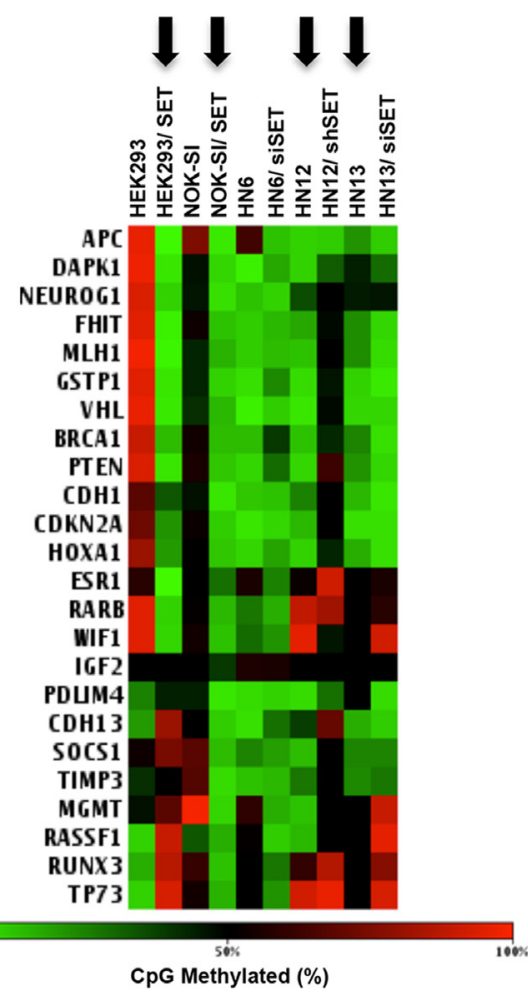

B

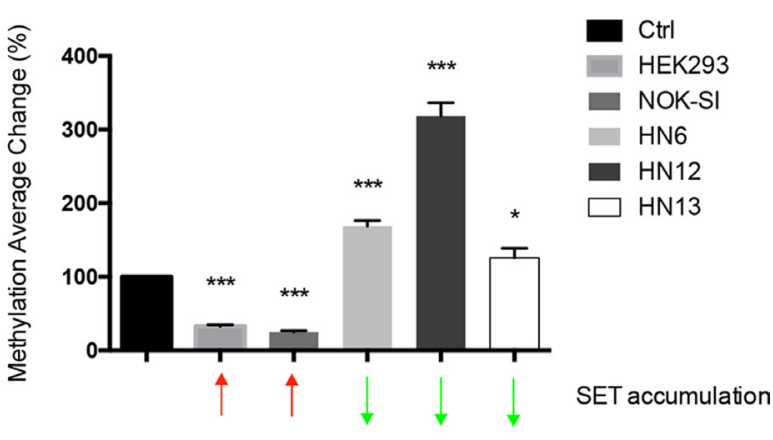

\section{C}
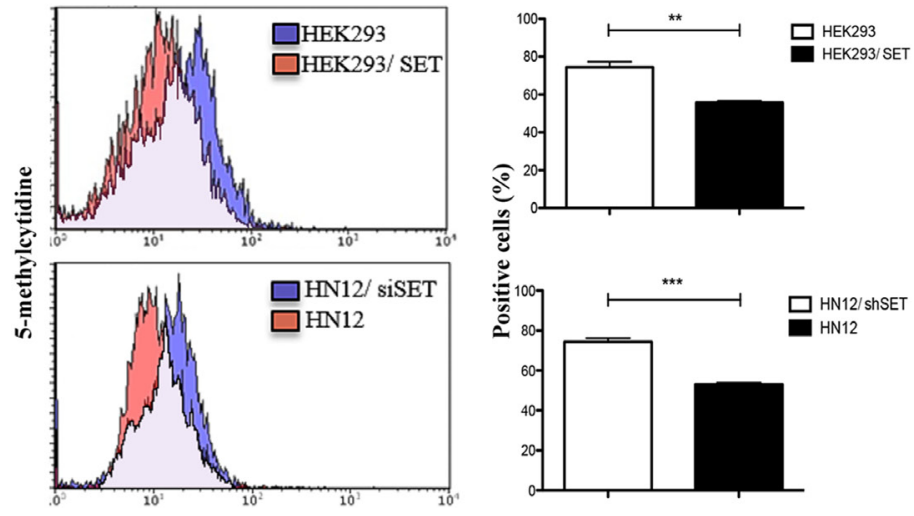

Figure 1: SET protein promotes loss of DNA methylation. (A) Normal cell lines HEK293 and NOK-SI-control and overexpressing SET protein (HEK293/SET and NOK-SI/SET) -, and HNSCC cell lines HN6, HN12, and HN13 - control and with SET knockdown (HN6siSET, HN12siSET, and HN13siSET, respectively) - were used to assess the methylation profile of tumor suppressor genes. An empty vector and the siControl AllStar siRNA negative control oligonucleotide were used as controls for SET overexpression and SET knockdown, respectively. DNA was extracted with phenol:chloroform and the DNA methylation profile was assessed using EpiTect Methyl PCR array (Qiagen), according to the manufacturer's instructions. After qPCR assay, data were analyzed using PCR array Data Analysis Software (Qiagen). The heat map indicates hypomethylated genes in green and hypermethylated genes in red. (B) Graphic representation of the methylation average changes in cells after SET knockdown or overexpression. We calculated the ratio of changes in CpG methylation levels between control and SET up- or downregulated samples. (C) Flow cytometry assay was used to quantify global methylation levels though 5-methylcytidine accumulation in HEK293, HEK293/SET, HN12, and HN12siSET cells. Cells without antibody staining were used as negative control (no staining). Assays were performed in triplicate. ${ }^{*}(p<0.05),{ }^{* *}(p<0.01)$, and $* * *(p<0.001)$. 


\section{Active DNA demethylation is the mechanism activated by SET for the loss of methylation}

DNMTs are responsible for the establishment and maintenance of DNA methylation. In this regard, DNMT inhibition is the main mechanism of passive DNA demethylation [5]. Given that DNA methylation pattern is maintained through cell division by DNMT1 activation [25], we assessed whether SET overexpression reduces DNMT1 levels in HEK293, HN12, and HN13 cells, using an immunofluorescence assay. Remarkably, all SET-accumulating cells exhibited increased DNMT1 levels, and SET knockdown decreased DNMT1 levels (Figure 3A). As SET accumulation is associated with reduced DNA methylation, we would expect that SET overexpression downregulated DNMT1; then, we also
A

HEK293
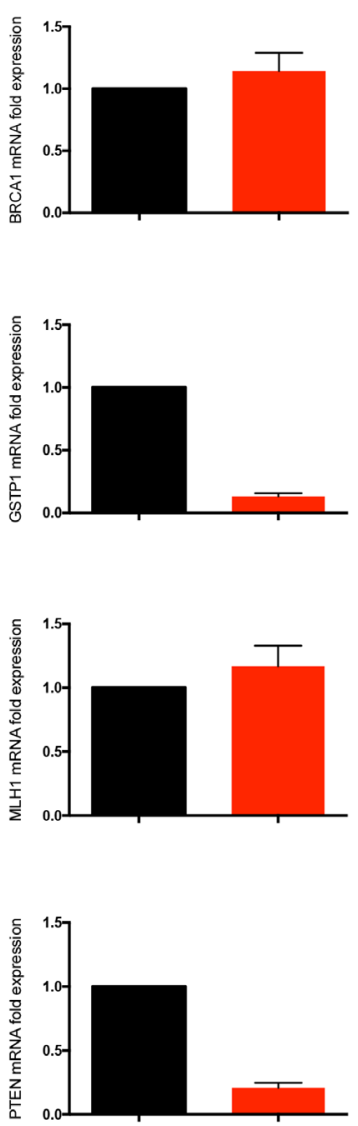

HN12
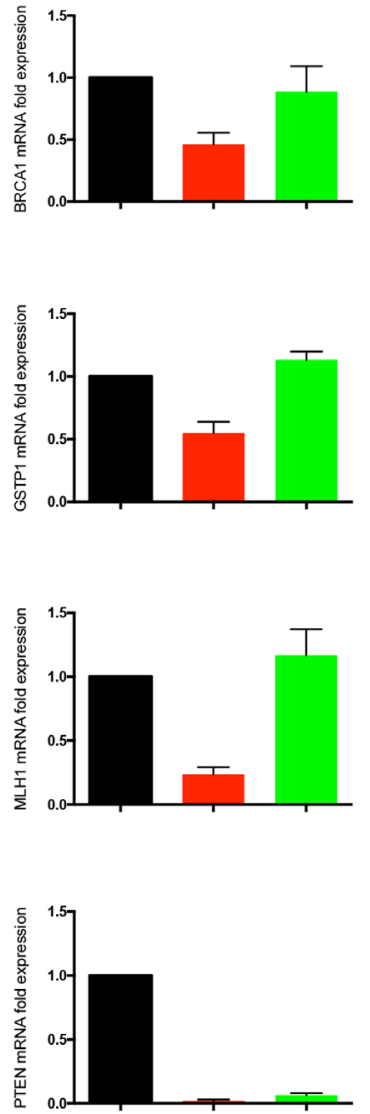

HN13
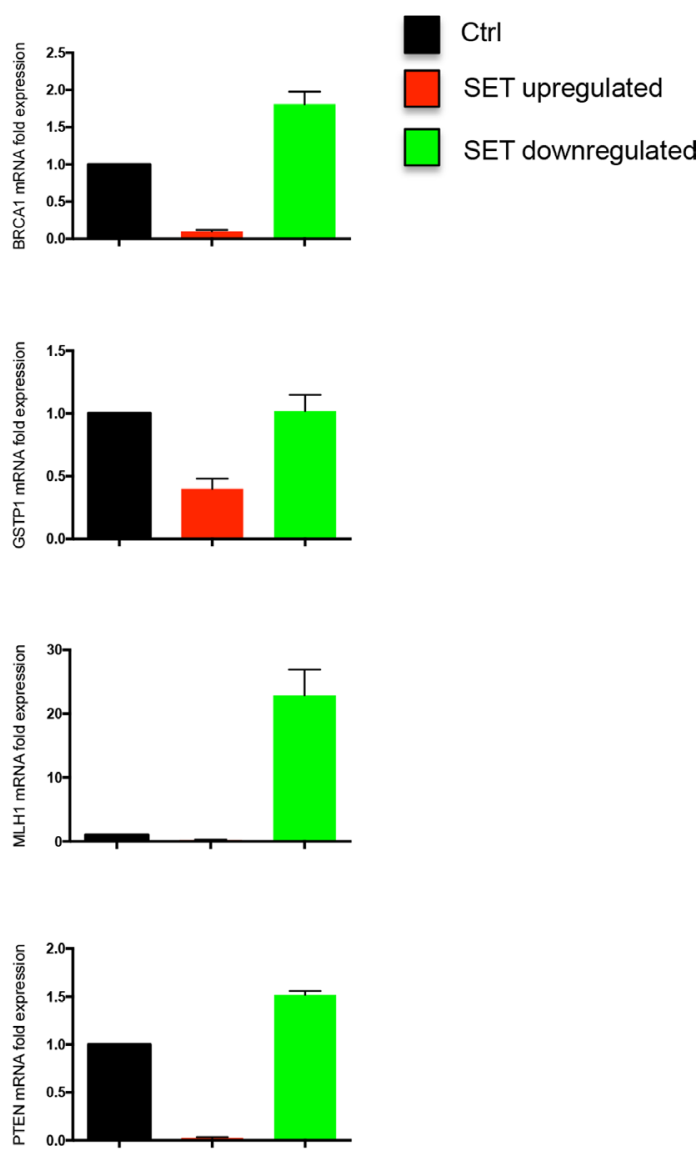

B

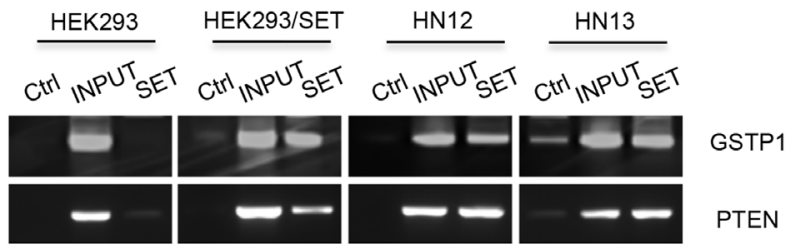

Figure 2: Loss of DNA methylation driven by SET protein does not necessarily activate gene expression. (A) Quantitative PCR was performed in HEK293, HEK293/SET, HN12, HN12siSET, HN13, and HN13siSET cells to evaluate whether tumor suppressor genes expression was affected by DNA methylation. BRCA1, MLH1, and PTEN were assessed through TaqMan probes and GSTP1 through SybrGreen primers. Graphics represent relative quantification of experiments performed in triplicate through $2^{-\Delta \Delta \mathrm{Ct}}$ method. CDNA from HEK293 was used as calibrator and GAPDH and $\beta$-globin primers were used as housekeeping. (B) Chromatin immunoprecipitation assay was performed to identify whether SET interacts with GSTP1 and PTEN promoters. Conventional PCR using DNA immunoprecipitated with antibody against SET was performed in triplicate. Ctrl lanes represent samples immunoprecipitated with anti-IgG antibody; INPUT samples consist of total DNA, and SET lanes refer to DNA immunoprecipitated with anti-SET antibody. 
assessed DNMT activity using the same cell lines. Accordingly, SET accumulation and downregulation were associated with augmented and diminished DNMT activity, respectively (Figure 3B).

DNA demethylation can also occur through an active mechanism. Active DNA demethylation is an enzymatic process that removes the methyl group from 5-methylcytosine by breaking carbon-carbon bonds [26]. One of the active DNA demethylation mechanisms involves the oxidation of 5-methylcytosine, which is converted into 5-hydroxymethylcytosine by TET enzymes [6]. Here, we used an immunofluorescence assay to determine the TET1 protein levels in HEK293, HN12, and HN13 cells. We detected high TET1 levels in SETaccumulating cells and decreased TET1 levels after SET knockdown (Figure 4A).

Since active demethylation converts 5-methylcytosine into 5-hydroxymethylcytosine [5], we also explored this product by flow cytometry. To assess exclusively the effect of the SET protein in the process, we selected the non-

A

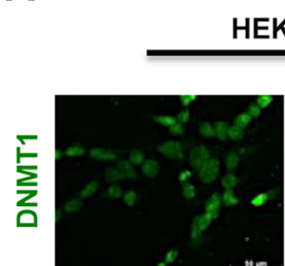

HEK293

HN12

HN13
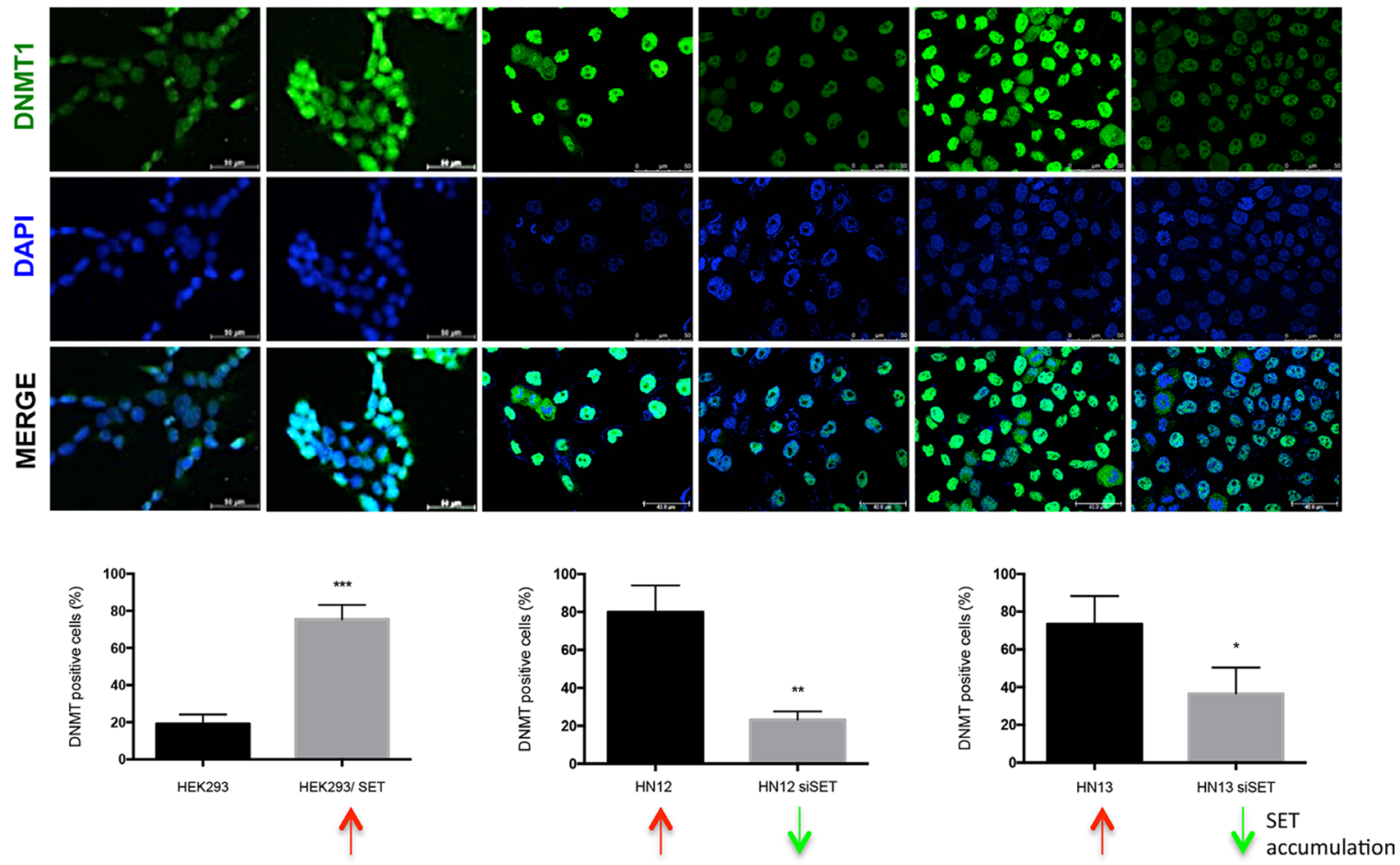

B

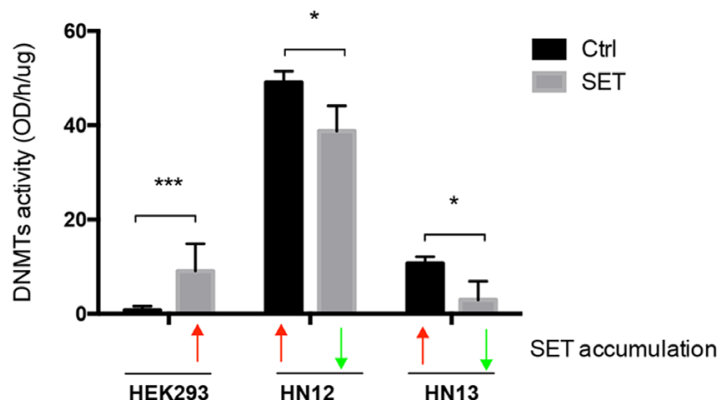

Figure 3: DNA methyltransferases are not involved in DNA demethylation process mediated by SET accumulation. (A) Immunofluorescence assay was performed in HEK293, HEK293/SET, HN12, HN12siSET, HN13, and HN13siSET cells to determine the levels of DNMT1, the enzyme responsible for DNA methylation maintenance. Cells from five fields were measured using ImageJ software. Graphics represent the percentage of DNMT1-positive cells. Red arrows indicate SET accumulation; green arrows indicate SET downregulation. (B) DNA methyltransferases enzymatic activity assay using nuclear protein extract from cells; this assay was perfomed using EpiSeeker DNMT Activity Quantification Kit (Abcam), according to the manufacturer's protocol. Graphic represents experiments performed in triplicate. ${ }^{*}(p<0.05),{ }^{* *}(p<0.01)$ and $* * *(p<0.001)$. 
tumour cell line HEK293 overexpressing it (Figure 4B). Consistent with the TET1 levels, 5-hydroxymethylcytosine levels increased in HEK293/SET cells, suggesting that SET accumulation promotes active DNA demethylation by elevating the levels of TET1, which subsequently controls the oxidation of 5-methylcytosine and its conversion into 5-hydroxymethylcytosine.

\section{SET accumulation downregulates gene expression through a histone deacetylation-dependent mechanism}

Although our finding have evidenced that SET promotes DNA demethylation through a wellcharacterized active mechanism, involving TET1

A
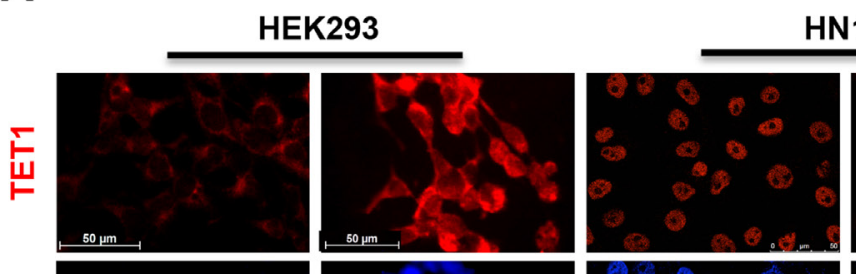

HN12
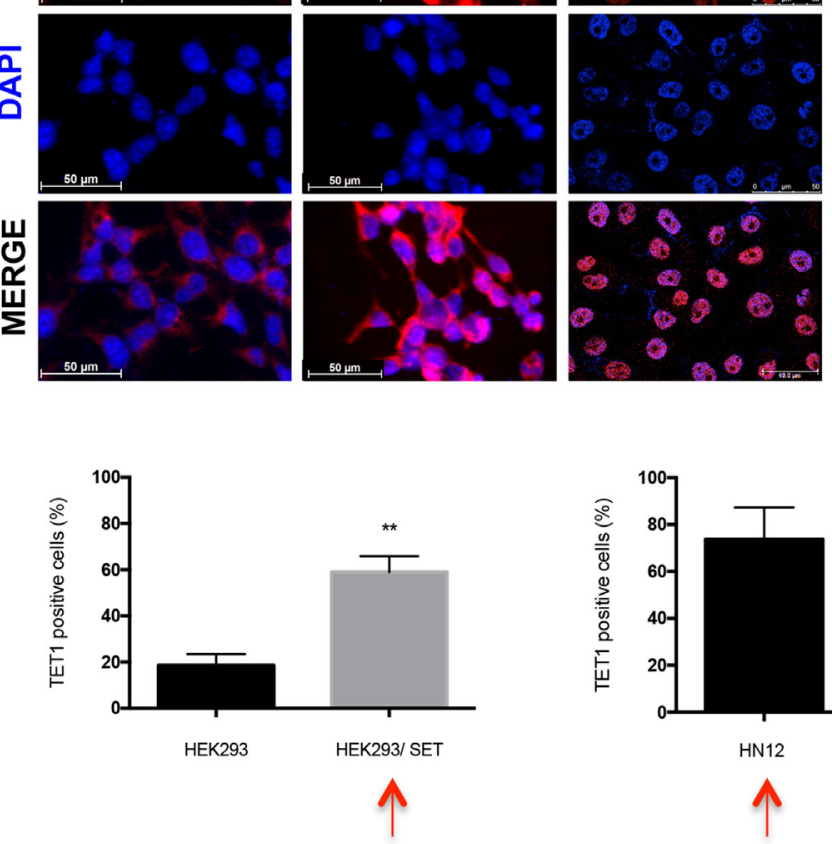

B
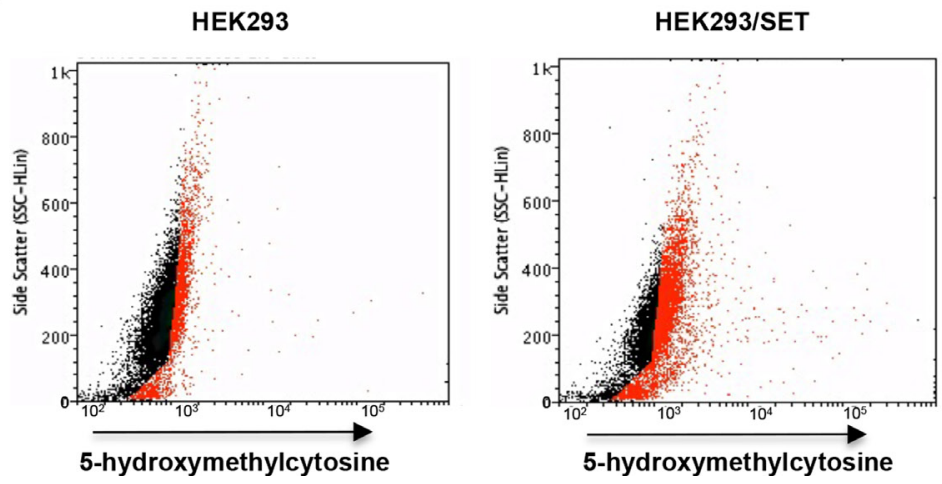
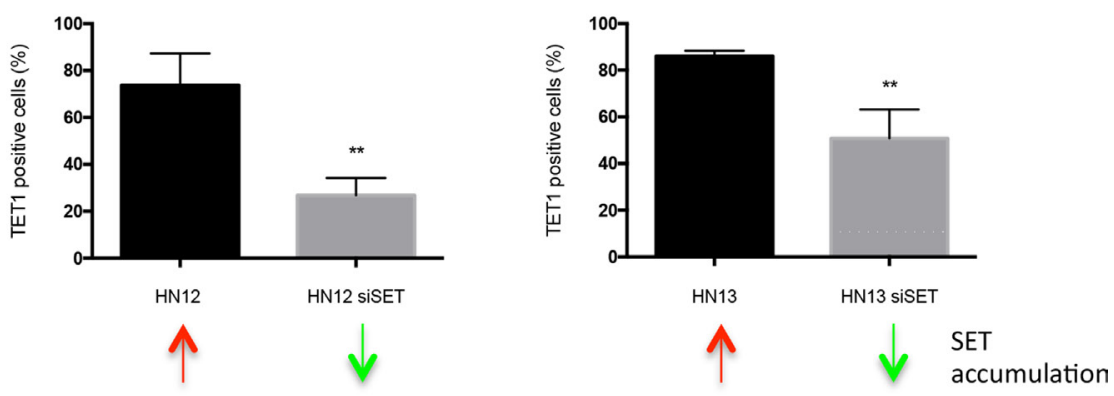
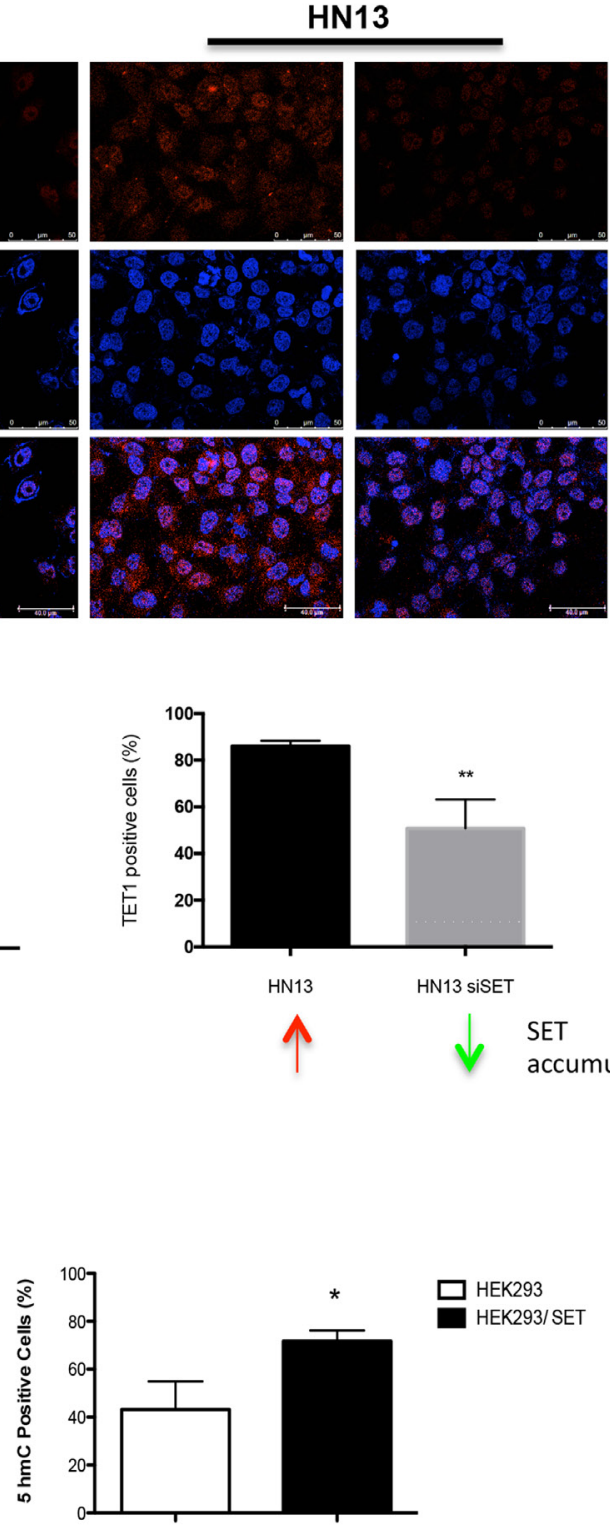

Figure 4: Loss of DNA methylation is associated with increased levels of TET1 and 5-hydroxymethylcytosine. (A) Immunofluorescence assay using anti-TET1 antibody was performed in HEK293, HEK293/SET, HN12, HN12siSET, HN13, and HN13siSET cells to assess active DNA demethylation process. Cells from five fields were measured using ImageJ software. Graphics represent the percentage of TET1-positive cells. Red arrows indicate SET accumulation; green arrows indicate SET downregulation. (B) Flow cytometry assay was performed to measure the levels of 5-hydroxymethylcytosine in HEK293 and HEK293/SET cells. The respective unstained cells were used as negative controls; dead cells were excluded from the plot. The assay was performed in triplicate. $*(p<0.05)$ and $* *(p<0.01)$. 
(Figure 4A) and 5-hydroxymethylcytosine (Figure 4B) [5], the present study also evidenced a downregulated gene expression driven by SET accumulation (Figure 2A). SET is a member of the INHAT complex that inhibits gene transcription through association with histones, thus preventing their acetylation by blocking the association histones-transcriptional coactivators [24]. To confirm the effects of SET accumulation on gene expression regulation, we performed a screening in HEK293 and HEK293/SET cells by employing a qPCR array system designed for a panel of 84 transcription factors (Table 1). We found that $42 \%$ of the genes were downregulated and $19 \%$ were upregulated. For validation of the results, we performed qPCR for 8 genes, whose expressions were altered, in HEK293, HEK293/SET, HN12, HN12siSET, HN13, and HN13siSET cells (Figure 5). SET accumulation downregulated six genes (ATF2, CTNNB1, HIF-1A, NFATC3, RELA, and STAT3) and upregulated two genes (ATF3 and MYB) (Figure 5 red bars). Overall, SET knockdown promoted the opposite effect (Figure 5 - green bars). We also assessed the effects of SET accumulation on the regulation of expression via promoters of two transcription factors, $N F k B$ and STAT3, by using a luciferase reporter assay. $N F k B$, which was upregulated as assessed by qPCR array analysis, presented increased levels in SET-accumulating cells (Figure 6A above). In agreement with the qPCR array results, STAT3 presented decreased levels in SET-accumulating cells (Figure 6A, below). These results confirm our previous findings that augmented SET levels control the expression of genes associated with tumorigenesis in HNSCC.

Next, we assessed whether protein levels of the transcription factors regulated by SET correlate with their respective mRNA levels. Western blotting analysis of six transcription factors (ATF1, CREB, NFkB, NFATC3, STAT1, and STAT3) demonstrated the existence of such correlation. In line with the gene expression data, the ATF1, CREB, and NFkB protein levels were elevated, and the NFATC3, STAT1, and STAT3 protein levels were diminished in SET-accumulating cells (Figure 6B).

Genes in an inactive state exhibit promoters that are hypoacetylated and allow SET binding to chromatin, thereby blocking the access of histone acetyltransferases and preventing gene expression [24]. Given that $42 \%$ of the transcription factors were downregulated in HEK293/ SET cells, we assessed whether SET protein interacts with chromatin to downregulate their expression. We performed the ChIP assay for the promoter region of HIF-1A and $N F A T C 3$, which were downregulated by SET (Figure 5), and of $N F k B$, which was upregulated by SET (Figure 6A). In SET-accumulating cells (HEK293/SET, HN12, and HN13), SET interacted with HIF-1A and NFATC3 chromatin, but not with $N F k B$ chromatin (Figure 6C).

SET binds to hypoacetylated histones and interacts with histone deacetylases, inhibiting the access of histone acetyltransferases to chromatin [13]. In this context, we assessed by Western blot whether SET accumulation changes the $\mathrm{H} 2 \mathrm{~B}$ and $\mathrm{H} 3$ acetylation levels (Figure 7A). Consistent with our previous report that SET decreases histone $\mathrm{H} 4$ acetylation [23], SET accumulation lowered both H2B and H3 acetylation levels, while SET knockdown elevated $\mathrm{H} 2 \mathrm{~B}$ and $\mathrm{H} 3$ acetylation; it suggests a mechanism of gene expression regulation mediated by SET that involves histone acetylation. As SET associates with class I and II histone deacetylases (HDACs) at deacetylated promoters to prevent histone acetylation and arrest gene expression [24], next, we used an immunofluorescence assay to assess the effects of SET accumulation on histone deacetylase 1 (HDAC1). As expected, SET accumulation and SET knockdown were associated with augmented and diminished HDAC1 levels, respectively (Figure 7B).

To demonstrate the role that SET protein plays in the promoter silencing through its direct interaction with hypoacetylated histones, we selected the pharmacological HDAC inhibitor, TSA (Trichostatin A), to increase histone acetylation levels [27]. After SET overexpression, HEK293 cells were treated with TSA, and a ChIP assay was performed to evaluate the capability of SET-promoter interaction (Figure $7 \mathrm{C}$ ). We analysed the promoter of HIF-1A and NFATC3, which were downregulated by the SET-promoter interaction (Figure 6C). For both genes, TSA decreased the SET-promoter interaction (Figure 7C black arrow). Western blot analysis of the efficacy of the TSA treatment demonstrated that it elevated the levels of acetylated histones $\mathrm{H} 2 \mathrm{~B}$ and $\mathrm{H} 3$, and remarkably diminished the SET protein levels (Figure 7D).

\section{Epigenetic control of gene expression mediated by SET accumulation is more effective through mechanisms involving histone acetylation}

As demonstrated above, SET accumulation promotes DNA hypomethylation through a mechanism of active DNA demethylation involving increased TET1 and 5-hydroxymethylcytosine generation. Controversially, SET decreased histone acetylation levels and expression of transcription factor genes. DNA methylation and histone acetylation are the most studied epigenetic modifications in the context of gene expression. Methylation of gene promoters may trigger deacetylation of histones and hypoacetylated histones may sensitize to targeted DNA methylation [28]. In this sense, we assessed the relationship between SET protein and gene expression using two pharmacological agents, 5-aza-2'-deoxycytidine (5-AZA) and TSA, to inhibit DNA methylation and activate histone acetylation, respectively. We performed qPCR in HEK293, HEK293/SET, HN12, HN12siSET, HN13, and HN13siSET cells to determine how the loss of methylation and gain of acetylation affect the expression of genes previously identified as being regulated by SET protein. By using a relative quantification method [29], we assessed the potential of pharmacological agents to restore 
Table 1: Human transcription factors qPCR array performed in HEK293 and HEK293/SET cells

\begin{tabular}{|c|c|c|c|c|c|}
\hline Gene ID & $\begin{array}{c}m R N A \text { Fold } \\
\text { Expression }\end{array}$ & Expression Levels & Gene ID & $\begin{array}{c}m R N A \text { Fold } \\
\text { Expression }\end{array}$ & Expression Levels \\
\hline AR & 0.3528 & decreased & MEF2B & 0.7254 & ns \\
\hline ARNT & 1.0769 & ns & MEF2C & 0.9375 & ns \\
\hline ATF1 & 1.523 & ns & MYB & 5.3034 & increased \\
\hline ATF2 & 0.4435 & decreased & MYC & 0.3888 & decreased \\
\hline ATF3 & 8.6154 & increased & MYF5 & - & - \\
\hline ATF4 & 1.4814 & ns & MYOD1 & 1.4016 & ns \\
\hline CEBPA & 1.3537 & ns & NFAT5 & 0.4225 & decreased \\
\hline CEBPB & 0.3071 & decreased & NFATC1 & 0.2906 & decreased \\
\hline CEBPG & 1.5877 & ns & NFATC2 & 0.4404 & decreased \\
\hline CREB1 & 2.9627 & increased & NFATC3 & 0.4331 & decreased \\
\hline CREBBP & 2.5614 & increased & NFATC4 & 2.095 & increased \\
\hline CTNNB1 & 0.3136 & decreased & NFKB1 & 2.5437 & increased \\
\hline DR1 & 1.1072 & ns & NFYB & - & - \\
\hline $\mathrm{E} 2 \mathrm{~F} 1$ & 0.3455 & decreased & NR3C1 & 0.6359 & ns \\
\hline E2F6 & 2.1095 & increased & PAX6 & 0.9506 & ns \\
\hline EGR1 & 2.1095 & increased & POU2AF1 & - & - \\
\hline ELK1 & 1.0259 & ns & PPARA & 0.7056 & ns \\
\hline ESR1 & 1.4015 & $\mathrm{~ns}$ & PPARG & 2.2925 & increased \\
\hline ETS1 & 1.2032 & ns & RB1 & 1.8881 & ns \\
\hline ETS2 & 1.5125 & ns & REL & 0.4411 & decreased \\
\hline FOS & 1.2457 & ns & RELA & 0.4196 & decreased \\
\hline FOXA2 & 0.4375 & decreased & RELB & 2.4401 & decreased \\
\hline FOXO1 & 1.4711 & ns & SMAD1 & 0.4483 & decreased \\
\hline GATA1 & - & - & SMAD4 & 1.4112 & ns \\
\hline GATA2 & 0.4343 & decreased & SMAD5 & 5.1228 & increased \\
\hline GATA3 & 0.2012 & decreased & SMAD9 & 0.4887 & decreased \\
\hline GTF2B & 3.046 & increased & SP1 & 2.6517 & increased \\
\hline GTF2F1 & 1.4917 & ns & SP3 & 1.9412 & ns \\
\hline HAND1 & 0.4284 & decreased & STAT1 & 0.4872 & decreased \\
\hline HAND2 & 0.9246 & ns & STAT2 & 0.2826 & decreased \\
\hline HDAC1 & 1.6323 & ns & STAT3 & 0.4722 & decreased \\
\hline HIF1A & 0.4108 & decreased & STAT4 & 1.7739 & ns \\
\hline HNF4A & 1.4015 & ns & STAT5A & 0.3136 & decreased \\
\hline HOXA5 & 0.4311 & decreased & STAT5B & 3.2873 & increased \\
\hline HSF1 & 2.3245 & increased & STAT6 & 1.3918 & ns \\
\hline ID1 & 1.22 & ns & TBP & 0.4508 & decreased \\
\hline IRF1 & 0.4294 & decreased & TCF1 & - & - \\
\hline JUN & 0.4053 & decreased & TCF7L2 & 1.4015 & ns \\
\hline JUNB & 0.6359 & ns & TFAP2A & 0.3384 & decreased \\
\hline JUND & 0.4165 & decreased & TGIF1 & 2.4913 & increased \\
\hline MAX & 0.4313 & decreased & ТР53 & 0.4815 & decreased \\
\hline MEF2A & 0.6493 & ns & YY1 & 1.3076 & ns \\
\hline
\end{tabular}

mRNA fold change expression was calculated using PCR array Data Analysis Software (Qiagen). Genes with fold expression value below 0.5 were considered downregulated, and genes with fold expression value above 2.0 were considered upregulated. ns (non-significant). 
A
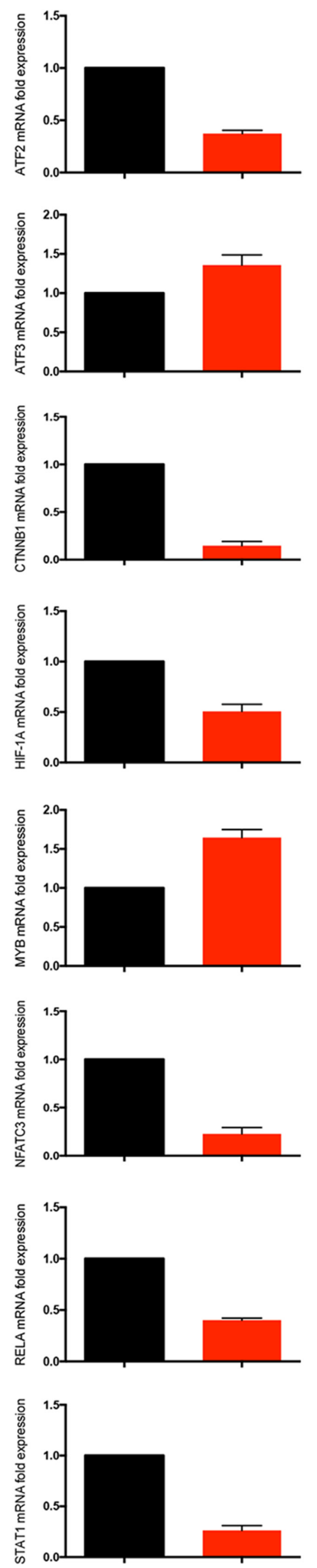

HN12
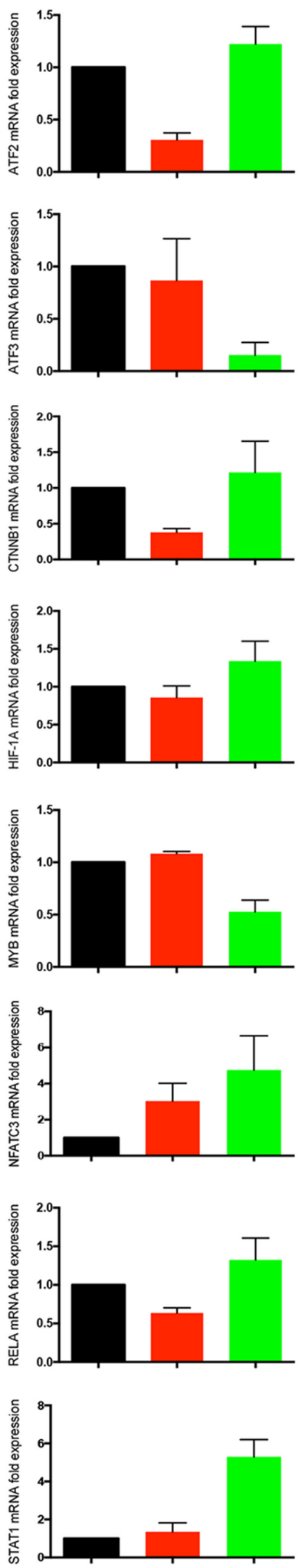

HN13
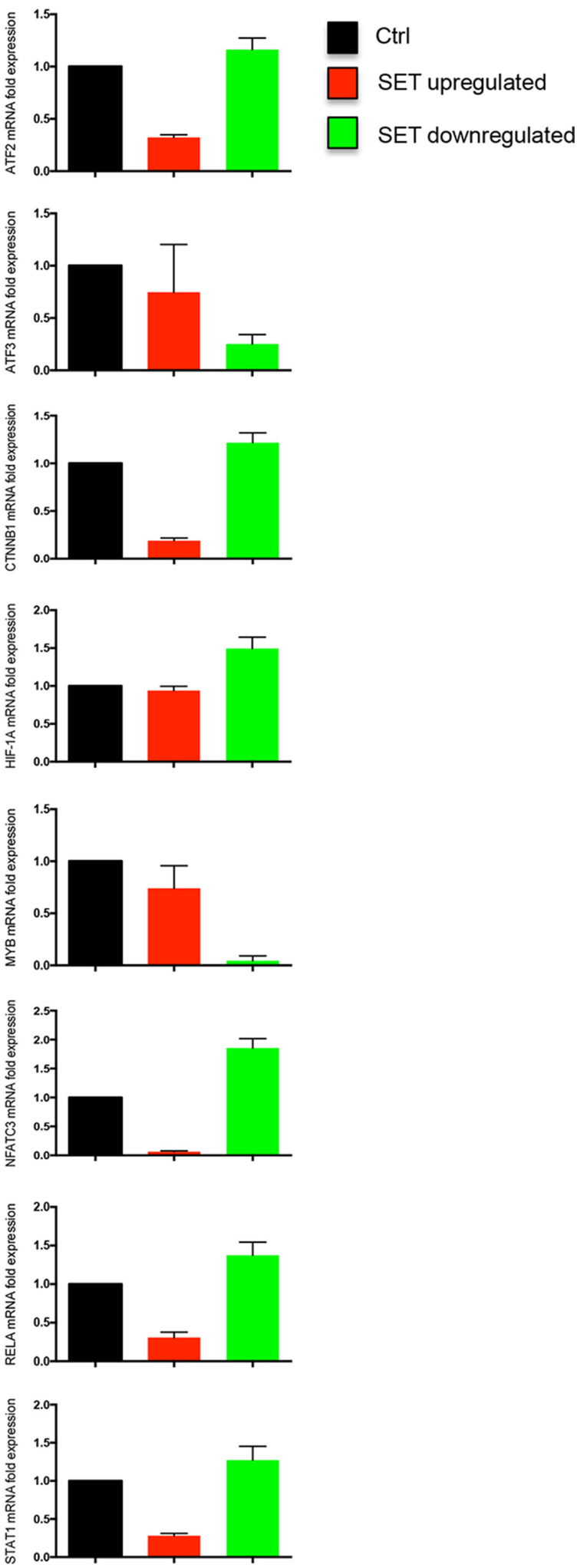

Figure 5: Gene expression of human transcription factors is downregulated through SET protein. Quantitative real time PCR was performed to validate the gene expression of six transcription factors downregulated by SET (ATF2, CTNNB1, HIF-1A, NFATC3, RELA, and STAT1) and two transcription factors upregulated by SET (ATF3 and MYB), using SybrGreen primers in HEK293, HEK293/ SET, HN12, HN12siSET, HN13, and HN13siSET cells. Graphics represent relative quantification of experiments performed in triplicate through the $2^{-\Delta \Delta \mathrm{Ct}}$ method. CDNA from HEK293 cells was used as calibrator; GAPDH and $\beta$-globin primers were used as housekeeping. 
A
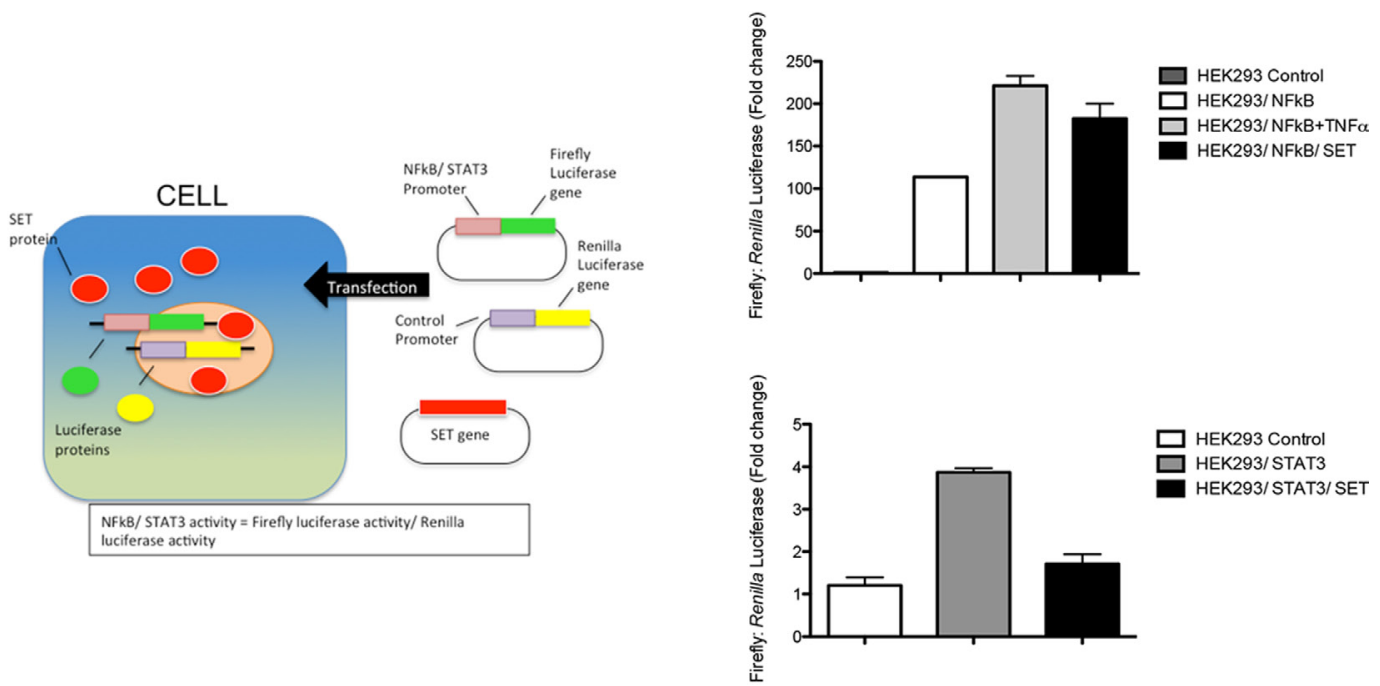

B

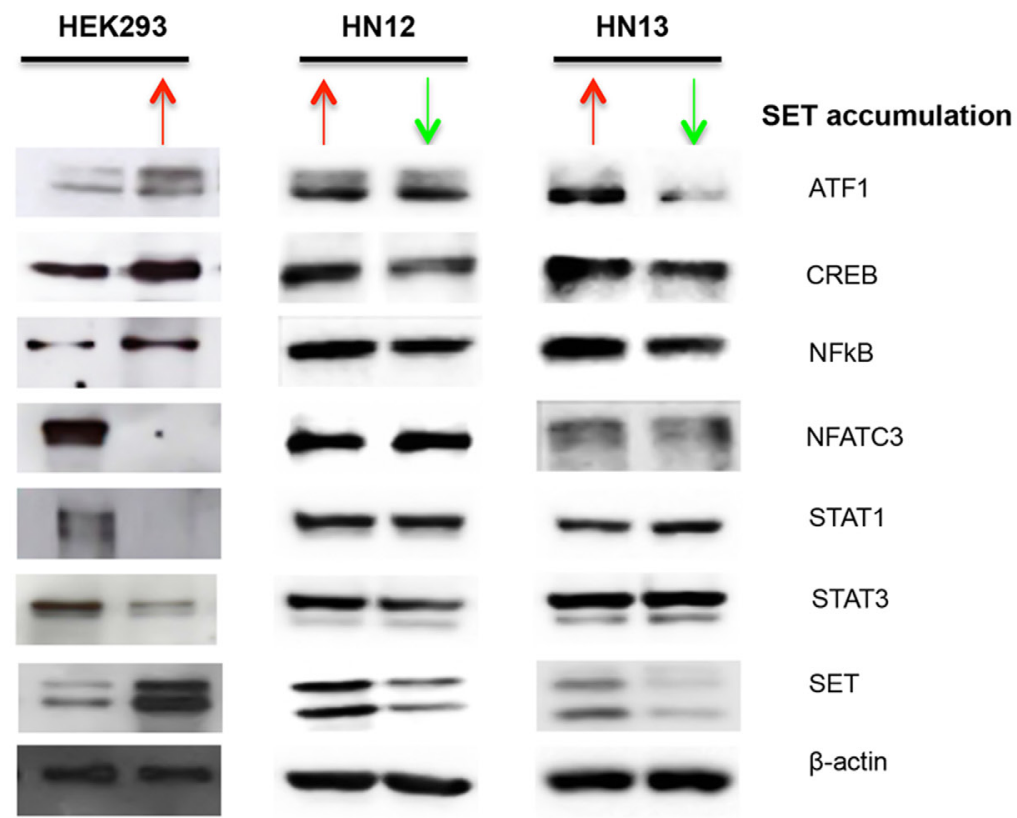

C

\begin{tabular}{|c|c|c|c|c|}
\hline HEK293 & HEK293/SET & HN12 & HN13 & \\
\hline$\therefore$ NPU & $C$ Cri N NPUT SET & $C+r \mid N P U T$ & $C$ Cri $N P U_{S}^{\top}{ }_{S T}$ & \\
\hline & & - & -- & HIF-1A \\
\hline - & $5=$ & 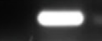 & $-\infty=$ & NFATC3 \\
\hline$\sigma$ & - & - & 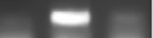 & NFkB \\
\hline
\end{tabular}

Figure 6: SET contributes to low gene expression levels through chromatin association. (A) Luciferase reporter assay was performed to quantify NFkB and STAT3 expression induced by SET accumulation. HEK293 cells were transfected with plasmids containing cDNA to overexpress SET, and plasmids with gene promoter region for NFkB (PGL3-NFkB-Luc) and STAT3 (PGL3-STAT3-Luc). (B) Western blot assay was used to assess whether downregulation of gene expression could be controlling protein levels. Total proteins were obtained from HEK293, HEK293/SET, HN12, HN12siSET, HN13, and HN13siSET cells. ATF1, CREB, NFkB, NFATC3, STAT1, STAT3, SET, and $\beta$-actin proteins were analyzed. Red arrows indicate SET accumulation; green arrows indicate SET downregulation. (C) Chromatin immunoprecipitation assay was performed to assess whether SET is able to downregulate the expression of transcription factor genes through binding to the promoter. DNA of the respective cells was immunoprecipitated with anti-SET antibody, and PCR was performed using primers for HIF-1A, NFATC3, and NFkB promoters. Ctrl lanes represent samples immunoprecipitated using anti-IgG antibody; INPUT samples consist of total DNA; SET lanes refer to DNA immunoprecipitated with anti-SET antibody. 
A
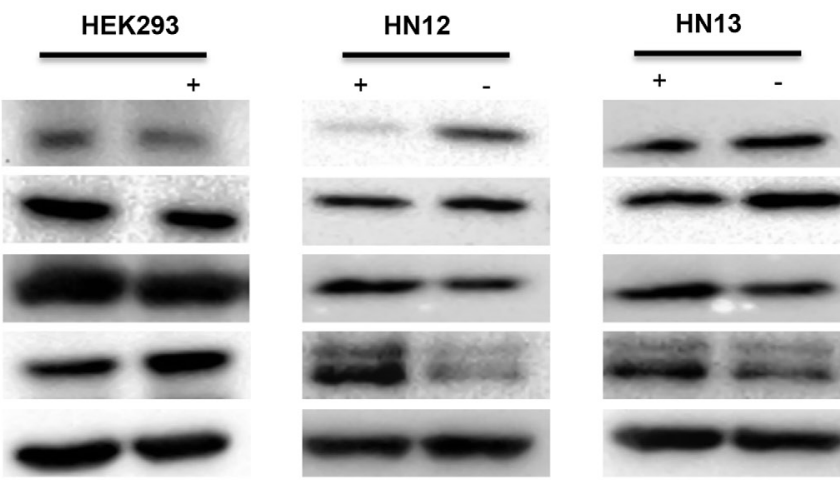

SET

acetyl-H2B

acetyl-H3

histone-H3

SET

$\beta$-actin

B
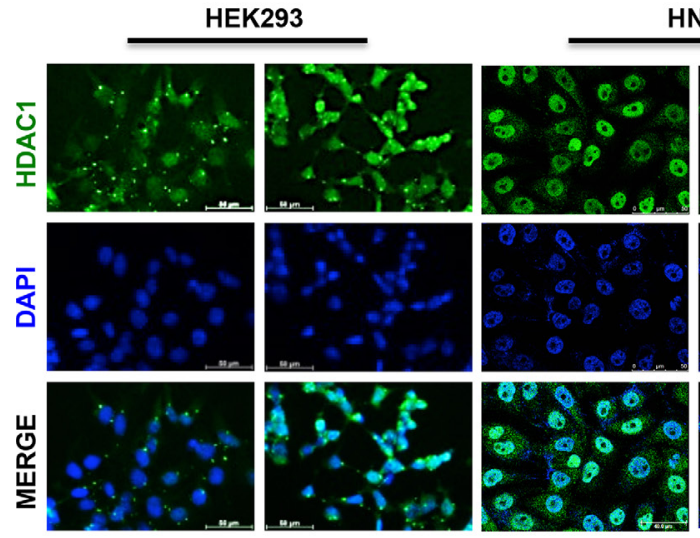

HN12
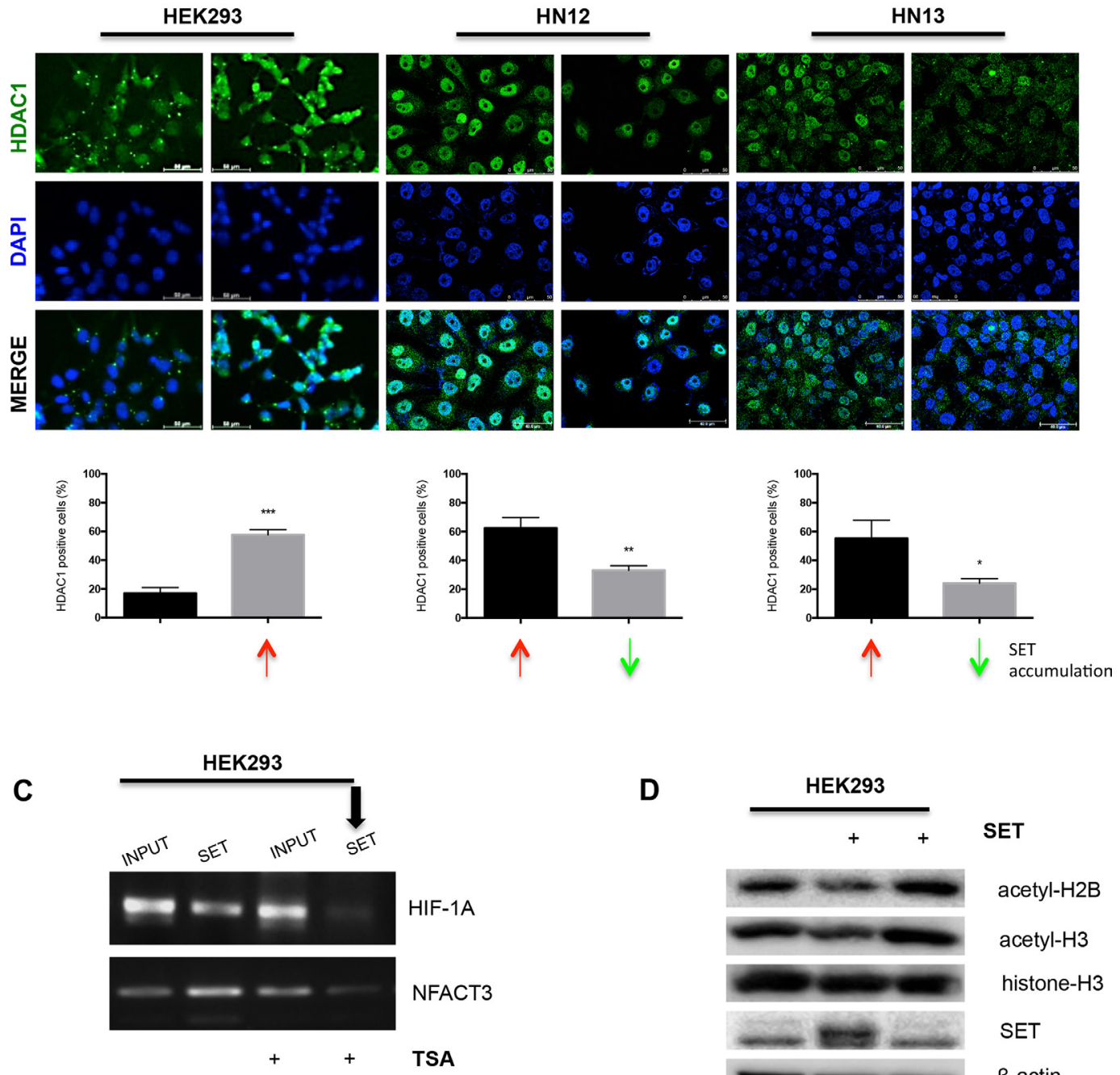

D

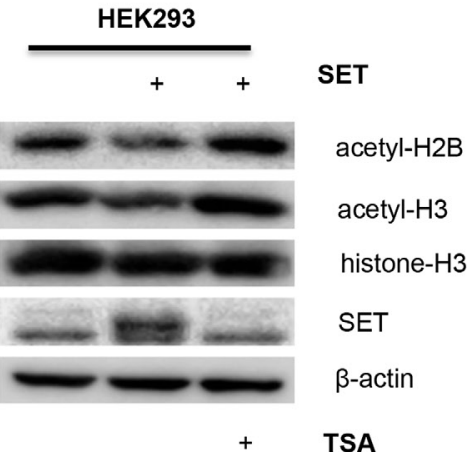

Figure 7: SET may modulate gene expression through hypoacetylation of histones and increase of HDAC1 levels. (A) Western blot assay was performed by adding antibodies against acetyl-H2B, acetyl-H3, SET, and $\beta$-actin to total proteins extracted from HEK293, HEK293/SET, HN12, HN12siSET, HN13, and HN13siSET cells. (B) HDAC1 levels were assessed using immunofluorescence assay. Cells from five fields were measured using ImageJ software. Graphics represent the percentage of TET1-positive cells. Red arrows indicate SET accumulation; green arrows indicate SET downregulation. (C) To assess whether SET directly associates with sites of hypoacetylated gene promoter, HEK293 and HEK293/SET cells were treated with the histone deacetylase inhibitor Trichostatin A (TSA; $100 \mathrm{ng} / \mathrm{mL}$ ) for $24 \mathrm{~h}$. After cell lysis, chromatin was immunoprecipitated with anti-SET antibody and PCR was performed using primers that recognize the promoter of HIF-1A and NFATC3. INPUT samples consist of total DNA; SET lanes refer to DNA immunoprecipitated with anti-SET antibody. (D) Western blot assay using antibodies against acetyl-H2B, acetyl-H3, SET, and $\beta$-actin was performed with total proteins from the respective cells treated with TSA $(100 \mathrm{ng} / \mathrm{mL})$ for $24 \mathrm{~h}$. 
the gene expression levels close to those observed in cells without SET accumulation. In general, TSA was more effective than 5-AZA to reverse the effect of SET protein accumulation on gene expression (Figure 8A). Therefore, SET accumulation likely represses gene expression through a histone hypoacetylation-associated mechanism.

Regulation of gene expression depends on histone modifications, such as histone acetylation/methylation, DNA methylation, chromatin remodeling enzymes and effector proteins that, in association, influence the structure and function of chromatin [30]. In addition, evidence suggests an interplay between DNA methylation and histone deacetylation in the silencing of gene transcription [28]. Given that our results indicated that SET accumulation promotes DNA demethylation and histone deacetylation, as well as downregulation of genes, we assessed whether these epigenetic modifications, promoted by SET accumulation, could reflect modifications in chromatin organization. Transmission electron microscopy (TEM) images revealed that SETaccumulating HEK293 cells presented an increase in electron-dense material scattered by the nucleus, which was compatible with chromatin structure, despite exhibiting a loss of the heterochromatin associated with the nuclear lamina (Figure 8B). These results may explain how SET regulates gene expression levels though histone deacetylation and chromatin condensation: together, they could be promoting the repression of transcription.

\section{DISCUSSION}

SET protein, which was first described as stimulating DNA replication and transcription of the adenovirus genome protein [31], has been associated with multiple actions involved in chromatin remodeling and transcription control [10, 13, 32-34]. A SET function linking epigenetic modifications and gene expression has not yet been described. In this context, and considering that SET accumulates in HNSCC [9, 15], we assessed whether SET accumulation could interfere with epigenetic and gene expression control to contribute to cancer development. Here, we demonstrated that SET accumulation is associated with loss of DNA methylation, apparently through active DNA demethylation signaling and, remarkably, reduced methylation levels seem not to directly correlate with gene expression control.

HNSCC is a heterogeneous disease in which DNA methylation status changes according to clinical, environmental, and genetic factors [35]. DNA global hypomethylation has been reported in HNSCC and has been associated with tumour progression, regardless of the methylation status of the gene promoter regions [36]. As expected, we found that three HNSCC cell lines exhibited diminished DNA methylation when compared with normal oral keratinocyte spontaneously immortalized (NOK-SI) and HEK293 cells. SET protein knockdown through
RNA interference recovered the DNA methylation status, which was accompanied by augmented 5-methylcytidine levels, indicating that SET participates in the control of global methylation by promoting loss of methylation in HNSCC. However, loss of methylation was not followed by increased expression of the genes analysed. According to TEM images, loss of methylation resulting from SET accumulation may be associated with either reactivation of elements of peripheral heterochromatin, repetitive elements or oncogene activation. These are factors known to favour carcinogenesis [37, 38].

Loss of DNMT1 leads to progressive DNA demethylation through a passive mechanism [26, 39]. It is worth to note that SET accumulation increased both DNMT1 protein levels and enzymatic activity. This finding suggests that the DNA demethylation mechanism associated with SET accumulation is not mediated by DNMT1 loss in a passive manner [40]. DNMT1 is the major enzyme that maintains the cellular DNA methylation levels [41] but, in tumour cells, DNMT1 up-regulation is associated with cellular transformation in tumours presenting both global hyper- or hypomethylation [42-44].

One explanation for the loss of methylation promoted by SET involves the active DNA demethylation process, based on TET proteins that successively oxidize 5-methylcytosine to 5-hydroxymethylcytosine, 5-formylcytosine, and 5-carboxylcytosine, which in turn can be removed from DNA through a base excision repair mechanism $[40,45]$. Accordingly, we demonstrated that SET accumulation is associated with a rise in TET1 and 5-hydroxymethylcytosine levels, suggesting the involvement of SET in the active DNA demethylation through accumulation of TET1, which in turn drives 5-hydroxymethylcytosine formation.

Our initial hypothesis that global loss of methylation would result in increased expression of all studied genes was not confirmed; in fact, SET protein downregulated most of the genes analyzed. Recent studies have demonstrated that specific histone modifications can mediate gene repression in cancer cells in the gene promoter region through a DNA methylation stateindependent mechanism [46-48]. In addition, it has been proposed that, rather than a mechanism to stably lock gene expression, DNA methylation works as a marker to maintain gene silencing $[49,50]$. Surprisingly, SET accumulation did not increase the expression of the hypomethylated genes analyzed, suggesting that SET regulates gene expression through a mechanism independent of DNA methylation of the gene promoter regions. Given that SET accumulation inhibits histone acetylation [13], we assessed whether SET negatively regulates gene expression by controlling the histone acetylation process.

Lysine acetylation in histone tails relaxes the chromatin structure and favours gene transcription $[51,52]$. SET protein was identified in association with 
A
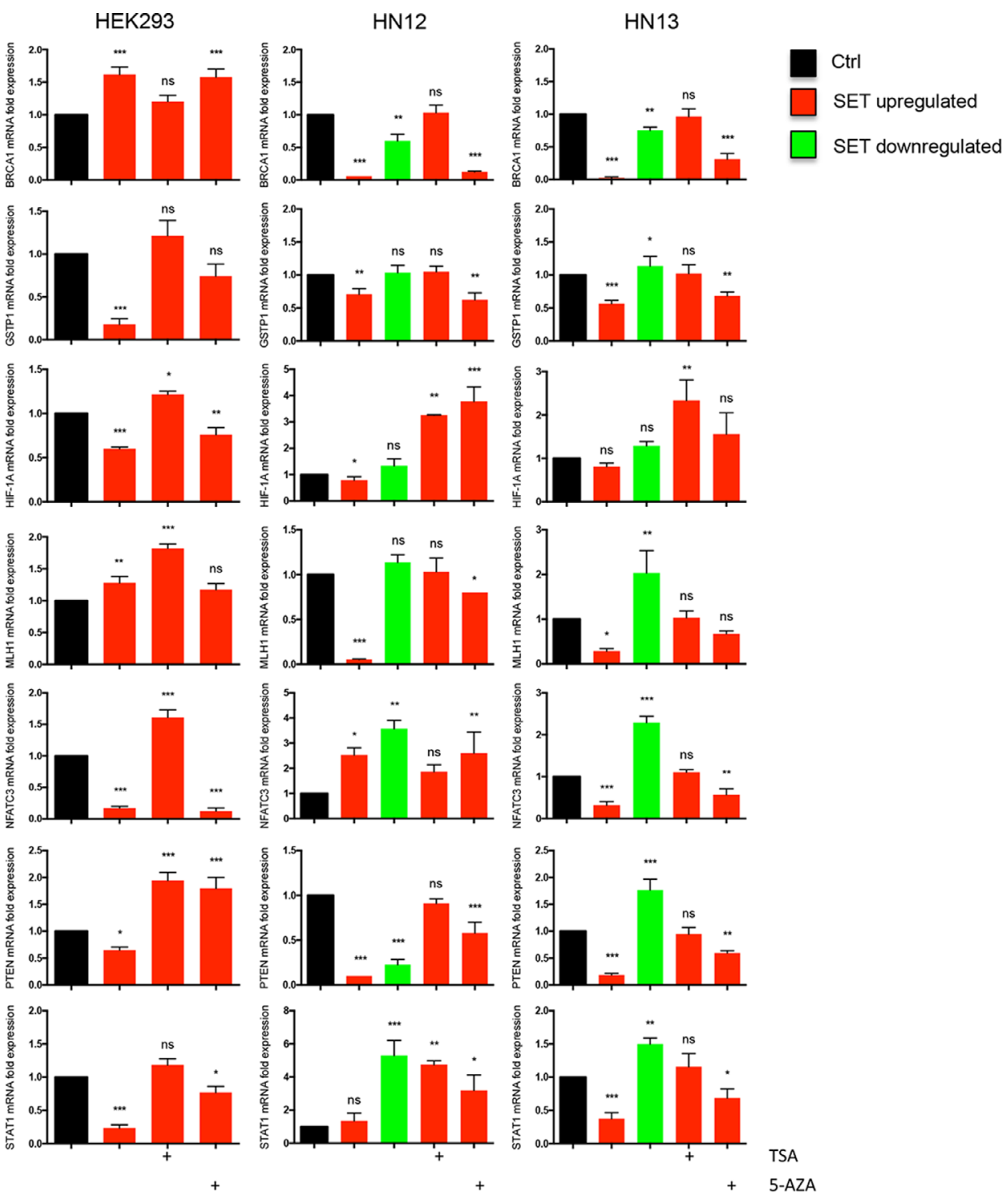

B

HEK293

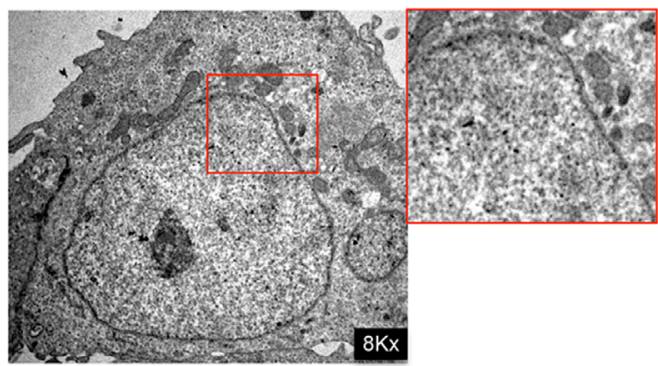

HEK293/ SET

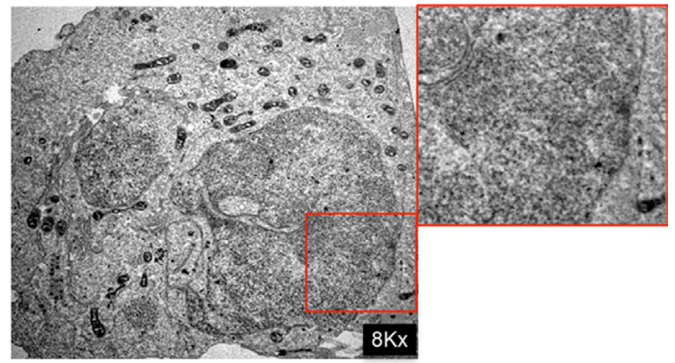

Figure 8: Histone acetylation is more effective than DNA demethylation in reactivating gene expression in SETaccumulating cells. (A) Quantitative real time PCR was performed using cDNA from HEK293, HEK293/SET, HN12, HN12siSET, HN13, and HN13siSET cells treated with $100 \mathrm{ng} / \mathrm{mL}$ TSA (histone deacetylase inhibitor) or $10 \mu \mathrm{M} 5$-aza-2'-deoxicytidine (DNA methyltransferase inhibitor). BRCA1, MLH1, and PTEN were assessed through TaqMan probes, and GSTP1, HIF-1A, NFATC3, and STAT1 through SybrGreen primers. Graphics represent relative quantification of experiments performed in triplicate, calculated using the $2^{-\triangle \Delta C t}$ method. CDNA from HEK293 cells was used as calibrator; GAPDH and $\beta$-globin primers were used as housekeeping. $*(p<0.05), * *(p<0.01), * * *(p<0.001)$ and ns (non-significant). (B) Transmission electron microscopy was performed in HEK293 and HEK293/SET cells to analyze chromatin compaction. 
hypoacetylated histones, maintaining the gene silencing $[23,24]$. Here, we found that SET accumulation lowered the expression of most of the genes analysed, regardless of DNA methylation state. Given that histone deacetylase inhibitors can reactivate gene expression without loss of methylation [50], we used TSA, a pharmacological HDAC inhibitor, to increase histone acetylation and 5-AZA, a demethylating agent, to inhibit DNA methylation. By exploring the gene expression levels, our results demonstrated that TSA was more effective than 5-AZA in recovering the gene expression close to basal levels, suggesting that (i) the main mechanism of gene transcription silencing involves histone hypoacetylation and chromatin compacting in SET-accumulating cells, and (ii) this mechanism is independent of the DNA methylation status.

This study provides evidence supporting that SET accumulation is important for tumorigenesis in HNSCC. SET would regulate the expression of several genes associated with cell differentiation, DNA damage repair, and development that we investigated, such as transcription factors and tumour suppressors (STAT1, STAT3, NFkB, NFATC3, MLH1, PTEN, BRCA1, HIF1A, $A T F 1$, and $C R E B$ ). Furthermore, DNA demethylation, associated with SET accumulation, may also favour HNSCC tumorigenesis by, for example, reactivating heterochromatin elements as repetitive sequences and oncogenes.

Overall, SET accumulation is implicated in epigenetic modifications, such as DNA hypomethylation and histone hypoacetylation. It is associated with gene expression regulation and chromatin organization, thereby affecting gene expression control, DNA damage repair, and genome instability. Certainly, SET protein activity can contribute to HNSCC development. We propose HDAC inhibitors as potential pharmacological agents to recovery gene expression in SET-accumulating tumours.

\section{MATERIALS AND METHODS}

\section{Cell lines and transfection}

Head and neck squamous cell carcinomas (HNSCCs) HN6, HN12, and HN13 [53], HEK293 (human embryonic kidney; ATCC, Manassas, VA, USA) and NOK-SI (normal oral keratinocytes) [54] cell lines were cultured in DMEM supplemented with $10 \%$ fetal bovine serum, penicillin $(100 \mathrm{U} / \mathrm{ml})$, and streptomycin $(100 \mathrm{mg} / \mathrm{ml})$ (SigmaAldrich, St. Louis, MO, USA), at $37^{\circ} \mathrm{C}$, in a humidified atmosphere containing $5 \% \mathrm{CO}_{2}$.

For assays using SET overexpression, HEK293 and NOK-SI cells were transfected with SET DNA construction using the PolyFect Reagent (Qiagen, Valencia, CA, USA) following the manufacturer's instructions: HEK293/SET and NOK-I/SET, respectively. A SET human full-length cDNA clone (pCMV SPORT6;
NM_003011.3) was purchased from Invitrogen and transferred to a pcDNA3.1 vector (Invitrogen - ThermoFisher Scientific - Waltham, MA, USA). Negative control cells were transfected using a pcDNA3.1 empty vector.

For assays using SET knockdown, HNSCC cell lines were transfected with double-stranded RNA oligonucleotides directed against SET (GS6418; Qiagen, Valencia, CA, USA) using HiPerFect Transfection Reagent (Qiagen, Valencia, CA, USA) following the manufacturer's instructions: HN6siSET, HN12siSET, and HN13siSET, respectively. The siCONTROL AllStars siRNA Negative Control (Qiagen, Valencia, CA, USA) was used as a negative siRNA control.

\section{DNA methylation analysis}

Genomic DNA was isolated from cell lines through digestion with proteinase $\mathrm{K}$ followed by phenol-chloroform extraction and ethanol precipitation. Genomic DNA $(1 \mu \mathrm{g})$ from HNSCC (control or with SET knockdown), HEK293 and NOK-SI (control or with SET overexpression) cells were digested using the EpitTect methyl DNA restriction Kit (Qiagen, Valencia, CA, USA) according to the manufacturer's instructions, and tumour suppressor gene EpiTect methyl qPCR Arrays (Qiagen, Valencia, CA, USA) were applied.

\section{Analysis of 5-methylcytidine and 5-hydroxymethylcytosine levels by using flow cytometry}

The protocol was performed as previously described [55]. Briefly, cells were washed twice with phosphate buffered saline (PBS) pH 7.4 supplemented with $1 \%$ bovine serum albumin (BSA) and $0.1 \%$ Tween 20 (PBSTBSA), and fixed with $0.25 \%$ paraformaldehyde. Cells were maintained at $4^{\circ} \mathrm{C}$ for $10 \mathrm{~min}$ before addition of $9 \mathrm{vol}$ of methanol/PBS. Then, cells were treated with $2 \mathrm{~N} \mathrm{HCl}$ at $37^{\circ} \mathrm{C}$ and with $0.1 \mathrm{M}$ borate buffer $\mathrm{pH}$ 8.5. Samples were treated with RNase $(10 \mathrm{mg} / \mathrm{mL})$, blocked with PBSTBSA and incubated with anti-5-methylcytidine (sc-56615 - Santa Cruz Biotechnology, Santa Cruz, CA, USA) or 5-hydroxymethylcytosine (ab214728 - Abcam, Cambridge, MA, USA). After incubation with TRITC-conjugated secondary antibody for $1 \mathrm{~h}$, cells were analysed using Guava easyCyte $^{\text {TM }}$ (Millipore, Billerica, MA, USA) cytometer.

\section{RNA extraction and real time PCR}

RNA was isolated from cell lines using TRIzol reagent (Invitrogen, Carlsbad, CA, USA), according to the manufacturer's protocol. RQ1 RNase-Free DNase (Promega, Madison, WI, USA) was used to treat RNA. cDNA synthesis was performed using a SuperScript III Reverse Transcriptase (Invitrogen, Carlsbad, CA, USA) protocol. 
The mRNA sequences were obtained from NCBI database (www.ncbi.nlm.nih.gov), and primers (Supplementary Table 1) were constructed using the Gene Runner program (version 3.05, Hastings Software, Inc.). Reactions were performed in a medium $(10 \mu \mathrm{L})$ containing $5 \mu \mathrm{L}$ Fast EvaGreen Master Mix (Uniscience, Cambridge, U.K.), $0.3 \mu \mathrm{M}$ per primer, and $100 \mathrm{ng}$ cDNA in a Mastercycler ep-Realplex thermocycler (Eppendorf, Hamburg, Germany). BRCA1 (Hs01556193_m1), MLH1 (Hs00979919_m1) and PTEN (Hs02621230_s1) mRNA levels were assessed by TaqMan assay (Thermo Fisher Scientific, Waltham, MA, USA) according to the manufacturer's protocol.

\section{Immunofluorescence assay}

Cells were placed on glass coverslips in 24-well plates and fixed with methanol for $6 \mathrm{~min}$, at $-20^{\circ} \mathrm{C}$. In addition, $0.5 \%(\mathrm{v} / \mathrm{v})$ Triton X-100 in PBS and 3\% (w/v) BSA was used for blocking. Primary antibodies antiDNMT1 (\#D59A4) and HDAC1 (\#10E2) (Cell signaling, Danvers, MA, USA) and anti-TET1 (\#sc-163443 Santa Cruz Biotechnology, Santa Cruz, CA, USA) were incubated overnight. After incubation with an Alexa Fluor 546-, FITC- or TRITC-conjugated secondary antibody for $1 \mathrm{~h}$, the cells were stained with DAPI (Sigma-Aldrich, St Louis, MO, USA) and visualized using a Zeiss Axiovert 40 CFL Microscope and Zeiss AxioVision 4.8.2 software (Munich, German).

\section{DMNT activity assay}

Protein fractions were obtained using ProteoJETTM Cytoplasmic and Nuclear Protein Extraction Kit (Fermentas - Thermo-Fisher Scientific - Waltham, MA, USA), according to the manufacturer's instructions. Nuclear fractions were subjected to a DNA methyltransferase activity assay using EpiSeeker DNMT Activity Quantification Kit (Abcam, Cambridge, MA, USA) according to the manufacturer's protocol.

\section{Human transcription factor PCR array}

Total RNA was isolated from cell lines using TRIzol reagent (Invitrogen, Carlsbad, CA, USA). Complementary DNA was synthetized using an $\mathrm{RT}^{2}$ first strand kit (SABioscience - Qiagen, Valencia, CA, USA). Real time PCR was performed using an $\mathrm{RT}^{2}$ Profiler PCR array system (SABioscience - Qiagen, Valencia, CA, USA) with SYBR Green PCR Master Mix, according to manufacturer's instructions, in a Mastercycler ep-Realplex thermocycler (Eppendorf, Hamburg, Germany).

\section{Luciferase reporter assay}

The protocol was performed as previously described [56]. Briefly, HEK293 cells were transfected with SET
pcDNA3.1 and pGL3-NFкB-Luc or pGL3-STAT3-Luc (kindly provided by Dr. Squarize) [56] promoter reporter constructs containing firefly luciferase cDNA, and pRLnull normalization construct containing Renilla luciferase from Renilla reniformis. Firefly and Renilla luciferase activity were measured in the cellular extract using DualGlo $^{\circledR}$ Luciferase Assay System (Promega, Madison, WI, USA), $24 \mathrm{~h}$ after transfection in a luminometer (AutoLumat LB 953, Berthold).

\section{Chromatin immunoprecipitation assay (ChIP)}

ChIP assay was performed as previously described [23]. Cells were cross-linked with $1 \%$ formaldehyde and then lysed. The DNA-protein complexes were immunoprecipitated with anti-SET (Santa Cruz Biotechnology, Santa Cruz, CA, USA) and protein G agarose beads (GE HealthCare, Piscataway, NJ, USA). DNA was extracted with phenol/chloroform post-reversing of the DNA-protein crosslinks. PCR reaction was performed using the sequence primers for GSTP1, HIF1A, NFATC3, NFkB and PTEN (Supplementary Table 2). PCR products were analysed using $1.5 \%$ agarose gel.

\section{Immunoblotting assay}

Cultured cells were harvested and sonicated, and protein concentration of the cellular extract was determined through DC protein assay (Bio-Rad, Hercules, CA, USA). Aliquots of total protein (30-50 $\mu \mathrm{g}$ ) from each sample were resolved by sodium dodecyl sulphate (SDS) PAGE and transferred to a PVDF membrane. The membrane was incubated overnight with primary antibodies for ATF1 (\#ab208436 - Abcam, Cambridge, MA, USA), CREB (\#9197), acetyl-H2B (Lys20) (\#2571) acetyl-H3 (Lys9) (\#9649), Histone H3 (\#9715), p65/ NFkB (\#3034), NFATC3 (\#4998), STAT1 (\#9172), STAT3 (\#12640) (Cell signaling, Danvers, MA, USA), SET (sc-133138) and $\beta$-actin (sc-47778) (Santa Cruz Biotechnology, Santa Cruz, CA, USA). The membrane was washed and incubated with secondary antibodies conjugated with horseradish peroxidase for $1 \mathrm{~h}$. Bound antibodies were detected using an ECL Western blotting system (GE Healthcare, Little Chalfont, UK).

\section{Transmission electron microscopy}

HEK293 and HEK293/SET cells were collected after trypsin digestion, washed in fresh PBS ( $\mathrm{pH} 7.4$ ), fixed by immersion in glutaraldehyde, post-fixed in osmium tetroxide, stained in block with uranium acetate, dehydrated, and embedded in resin (Embed 812, EM Sciences). Ultrathin sections $(60 \mathrm{~nm}$ ) were collected on Pioloform (Ted Pella, Redding, CA) and carbon-coated single sloth grids and contrasted with uranyl acetate and lead citrate. Cells were examined on a ZEISS LEO 906 electron microscope. 


\section{Statistical analyses}

Statistical analyses were performed using GraphPad Prism software (version 5.0, USA). Student's $t$-test or ANOVA was used to examine the association between media and treatments. $P$ values $<0.05$ were considered significant.

\section{ACKNOWLEDGMENTS AND FUNDING}

This work was supported by FAPESP (research grants \# 2013/10898-4, 2013/08135-2, 2010/20384-0, and 2009/52228-0, and fellowships \# 2009/10783-7 and 2010/20536-4) and CNPq. The authors thank Cristiana Bernadelli Garcia for her technical assistance.

\section{CONFLICTS OF INTEREST}

The authors declare no conflicts of interest.

\section{REFERENCES}

1. Brait M, Sidransky D. Cancer epigenetics: above and beyond. Toxicol Mech Methods. 2011; 21:275-288.

2. Glazer CA, Chang SS, Ha PK, Califano JA. Applying the molecular biology and epigenetics of head and neck cancer in everyday clinical practice. Oral Oncol. 2009; 45:440-446.

3. Goll MG, Bestor TH. Eukaryotic cytosine methyltransferases. Annu Rev Biochem. 2005; 74:481-514.

4. Jones PA, Baylin SB. The epigenomics of cancer. Cell. 2007; 128:683-692.

5. Bhutani N, Burns DM, Blau HM. DNA demethylation dynamics. Cell. 2011; 146:866-872.

6. Delatte B, Deplus R, Fuks F. Playing TETris with DNA modifications. EMBO J. 2014; 33:1198-1211.

7. Reddy KL, Feinberg AP. Higher order chromatin organization in cancer. Semin Cancer Biol. 2013; 23:109-115.

8. Kouzarides T. Chromatin modifications and their function. Cell. 2007; 128:693-705.

9. Patel V, Hood BL, Molinolo AA, Lee NH, Conrads TP, Braisted JC, Krizman DB, Veenstra TD, Gutkind JS. Proteomic analysis of laser-captured paraffin-embedded tissues: a molecular portrait of head and neck cancer progression. Clin Cancer Res. 2008; 14:1002-1014.

10. Gamble MJ, Fisher RP. SET, PARP1 remove DEK from chromatin to permit access by the transcription machinery. Nat Struct Mol Biol. 2007; 14:548-555.

11. Gamble MJ, Erdjument-Bromage H, Tempst P, Freedman LP, Fisher RP. The histone chaperone TAF-I/ SET/INHAT is required for transcription in vitro of chromatin templates. Mol Cell Biol,. 2005; 25:797-807.

12. Leung JW, Leitch A, Wood JL, Shaw-Smith C, Metcalfe K, Bicknell LS, Jackson AP, Chen J. SET nuclear oncogene associates with microcephalin/MCPH1 and regulates chromosome condensation. J Biol Chem. 2011; 286:21393-21400.
13. Seo SB, McNamara $\mathrm{P}$, Heo S, Turner A, Lane WS, Chakravarti D. Regulation of histone acetylation and transcription by INHAT, a human cellular complex containing the set oncoprotein. Cell. 2001; 104:119-130.

14. Cervoni N, Detich N, Seo SB, Chakravarti D, Szyf M. The oncoprotein Set/TAF-1beta, an inhibitor of histone acetyltransferase, inhibits active demethylation of DNA, integrating DNA methylation and transcriptional silencing. J Biol Chem. 2002; 277:25026-25031.

15. Leopoldino AM, Squarize CH, Garcia CB, Almeida LO, Pestana CR, Sobral LM, Uyemura SA, Tajara EH, Silvio Gutkind J, Curti C. SET protein accumulates in HNSCC and contributes to cell survival: antioxidant defense, Akt phosphorylation and AVOs acidification. Oral Oncol. 2012; 48:1106-1113.

16. Farrell AS, Allen-Petersen B, Daniel CJ, Wang X, Wang Z, Rodriguez S, Impey S, Oddo J, Vitek MP, Lopez C, Christensen DJ, Sheppard B, Sears RC. Targeting inhibitors of the tumor suppressor PP2A for the treatment of pancreatic cancer. Mol Cancer Res. 2014; 12:924-939.

17. Li M, Makkinje A, Damuni Z. The myeloid leukemiaassociated protein SET is a potent inhibitor of protein phosphatase 2A. J Biol Chem. 1996; 271:11059-11062.

18. Hu X, Garcia C, Fazli L, Gleave M, Vitek MP, Jansen M, Christensen D, Mulholland DJ. Inhibition of Pten deficient Castration Resistant Prostate Cancer by Targeting of the SET - PP2A Signaling axis. Sci Rep. 2015; 5:15182.

19. Liu GP, Wei W, Zhou X, Zhang Y, Shi HH, Yin J, Yao XQ, Peng CX, Hu J, Wang Q, Li HL, Wang JZ. I(PP2A) regulates p53 and Akt correlatively and leads the neurons to abort apoptosis. Neurobiol Aging. 2012; 33:254-264.

20. Kelkar A, Deobagkar D. A novel method to assess the full genome methylation profile using monoclonal antibody combined with the high throughput based microarray approach. Epigenetics. 2009; 4:415-420.

21. Gasche JA, Hoffmann J, Boland CR, Goel A. Interleukin-6 promotes tumorigenesis by altering DNA methylation in oral cancer cells. Int J Cancer. 2011; 129:1053-1063.

22. Mosquera Orgueira A. Hidden among the crowd: differential DNA methylation-expression correlations in cancer occur at important oncogenic pathways. Front Genet. 2015; 6:163.

23. Almeida LO, Goto RN, Pestana CR, Uyemura SA, Gutkind S, Curti C, Leopoldino AM. SET overexpression decreases cell detoxification efficiency: ALDH2 and GSTP1 are downregulated, DDR is impaired and DNA damage accumulates. FEBS J. 2012; 279:4615-4628.

24. Kutney SN, Hong R, Macfarlan T, Chakravarti D. A signaling role of histone-binding proteins and INHAT subunits pp32 and Set/TAF-Ibeta in integrating chromatin hypoacetylation and transcriptional repression. J Biol Chem. 2004; 279:30850-30855.

25. Law JA, Jacobsen SE. Establishing, maintaining and modifying DNA methylation patterns in plants and animals. Nat Rev Genet. 2010; 11:204-220. 
26. Wu H, Zhang Y. Reversing DNA methylation: mechanisms, genomics, and biological functions. Cell. 2014; 156:45-68.

27. Xu WS, Parmigiani RB, Marks PA. Histone deacetylase inhibitors: molecular mechanisms of action. Oncogene. 2007; 26:5541-5552.

28. Vaissiere T, Sawan C, Herceg Z. Epigenetic interplay between histone modifications and DNA methylation in gene silencing. Mutat Res. 2008; 659:40-48.

29. Pfaffl MW. A new mathematical model for relative quantification in real-time RT-PCR. Nucleic Acids Res. 2001; 29:e45.

30. Miller JL, Grant PA. The role of DNA methylation and histone modifications in transcriptional regulation in humans. Subcell Biochem. 2013; 61:289-317.

31. Guo H, Reddy SA, Damuni Z. Purification and characterization of an autophosphorylation-activated protein serine threonine kinase that phosphorylates and inactivates protein phosphatase 2A. J Biol Chem. 1993; 268:11193-11198.

32. Karetsou Z, Martic G, Tavoulari S, Christoforidis S, Wilm M, Gruss C, Papamarcaki T. Prothymosin alpha associates with the oncoprotein SET and is involved in chromatin decondensation. FEBS Lett. 2004; 577:496-500.

33. Matsumoto K, Nagata K, Okuwaki M, Tsujimoto M. Histone- and chromatin-binding activity of template activating factor-I. FEBS Lett. 1999; 463:285-288.

34. Karetsou Z, Martic G, Sflomos G, Papamarcaki T. The histone chaperone SET/TAF-Ibeta interacts functionally with the CREB-binding protein. Biochem Biophys Res Commun. 2005; 335:322-327.

35. Dikshit RP, Gillio-Tos A, Brennan P, De Marco L, Fiano V, Martinez-Penuela JM, Boffetta P, Merletti F. Hypermethylation, risk factors, clinical characteristics, and survival in 235 patients with laryngeal and hypopharyngeal cancers. Cancer. 2007; 110:1745-1751.

36. Smith IM, Mydlarz WK, Mithani SK, Califano JA. DNA global hypomethylation in squamous cell head and neck cancer associated with smoking, alcohol consumption and stage. Int J Cancer. 2007; 121:1724-1728.

37. Richards EJ. Quantitative epigenetics: DNA sequence variation need not apply. Genes Dev. 2009; 23:1601-1605.

38. Kalverda B, Roling MD, Fornerod M. Chromatin organization in relation to the nuclear periphery. FEBS Lett. 2008; 582:2017-2022.

39. Bird A. DNA methylation patterns and epigenetic memory. Genes Dev. 2002; 16:6-21.

40. Huang Y, Rao A. Connections between TET proteins and aberrant DNA modification in cancer. Trends Genet. 2014.

41. Miremadi A, Oestergaard MZ, Pharoah PD, Caldas C. Cancer genetics of epigenetic genes. Hum Mol Genet. 2007; 16 Spec No 1:R28-49.

42. Baylin SB, Herman JG, Graff JR, Vertino PM, Issa JP. Alterations in DNA methylation: a fundamental aspect of neoplasia. Adv Cancer Res. 1998; 72:141-196.
43. Bakin AV, Curran T. Role of DNA 5-methylcytosine transferase in cell transformation by fos. Science. 1999; 283:387-390.

44. Subramaniam D, Thombre R, Dhar A, Anant S. DNA methyltransferases: a novel target for prevention and therapy. Front Oncol. 2014; 4:80.

45. Kohli RM, Zhang Y. TET enzymes, TDG and the dynamics of DNA demethylation. Nature. 2013; 502:472-479.

46. Fraga MF, Ballestar E, Villar-Garea A, Boix-Chornet M, Espada J, Schotta G, Bonaldi T, Haydon C, Ropero S, Petrie K, Iyer NG, Perez-Rosado A, Calvo E, et al. Loss of acetylation at Lys16 and trimethylation at Lys20 of histone H4 is a common hallmark of human cancer. Nat Genet. 2005; 37:391-400.

47. Kondo Y, Shen L, Cheng AS, Ahmed S, Boumber Y, Charo C, Yamochi T, Urano T, Furukawa K, KwabiAddo B, Gold DL, Sekido Y, Huang TH, et al. Gene silencing in cancer by histone $\mathrm{H} 3$ lysine 27 trimethylation independent of promoter DNA methylation. Nat Genet. 2008; 40:741-750.

48. Rose NR, Klose RJ. Understanding the relationship between DNA methylation and histone lysine methylation. Biochim Biophys Acta. 2014; 1839:1362-1372.

49. Issa JP. DNA methylation as a clinical marker in oncology. J Clin Oncol. 2012; 30:2566-2568.

50. Raynal NJ, Si J, Taby RF, Gharibyan V, Ahmed S, Jelinek J, Estecio MR, Issa JP. DNA methylation does not stably lock gene expression but instead serves as a molecular mark for gene silencing memory. Cancer Res. 2012; 72:1170-1181.

51. Bannister AJ, Kouzarides T. Regulation of chromatin by histone modifications. Cell Res. 2011; 21:381-395.

52. Zhang T, Cooper S, Brockdorff N. The interplay of histone modifications - writers that read. EMBO Rep. 2015; 16:1467-1481.

53. Yeudall WA, Crawford RY, Ensley JF, Robbins KC. MTS1/ CDK4I is altered in cell lines derived from primary and metastatic oral squamous cell carcinoma. Carcinogenesis. $1994 ; 15: 2683-2686$.

54. Castilho RM, Squarize CH, Leelahavanichkul K, Zheng Y, Bugge T, Gutkind JS. Rac1 is required for epithelial stem cell function during dermal and oral mucosal wound healing but not for tissue homeostasis in mice. PloS one. 2010; 5:e10503.

55. Habib M, Fares F, Bourgeois CA, Bella C, Bernardino J, Hernandez-Blazquez F, de Capoa A, Niveleau A. DNA global hypomethylation in EBV-transformed interphase nuclei. Exp Cell Res. 1999; 249:46-53.

56. Squarize CH, Castilho RM, Sriuranpong V, Pinto DS Jr. and Gutkind JS. Molecular cross-talk between the NFkappaB and STAT3 signaling pathways in head and neck squamous cell carcinoma. Neoplasia. 2006; 8:733-746. 